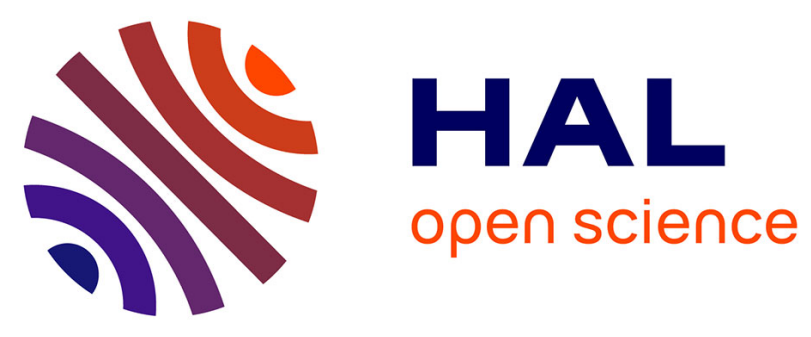

\title{
Cerebral neural correlates of differential melanopic photic stimulation in humans
}

Shao-Min P Hung, Dan P Milea, Annadata P Rukmini, Raymond P Najjar, Joo J Tan, Françoise Viénot, Marie Dubail, Sharon Lee Choon Tow, Tin P Aung, Joshua J Gooley, et al.

\section{To cite this version:}

Shao-Min P Hung, Dan P Milea, Annadata P Rukmini, Raymond P Najjar, Joo J Tan, et al.. Cerebral neural correlates of differential melanopic photic stimulation in humans. NeuroImage, 2017, 146, pp.763 - 769. 10.1016/j.neuroimage.2016.09.061 . hal-01565749

\author{
HAL Id: hal-01565749 \\ https://hal.science/hal-01565749
}

Submitted on 9 Aug 2017

HAL is a multi-disciplinary open access archive for the deposit and dissemination of scientific research documents, whether they are published or not. The documents may come from teaching and research institutions in France or abroad, or from public or private research centers.
L'archive ouverte pluridisciplinaire HAL, est destinée au dépôt et à la diffusion de documents scientifiques de niveau recherche, publiés ou non, émanant des établissements d'enseignement et de recherche français ou étrangers, des laboratoires publics ou privés. 


\section{Cerebral Neural Correlates of Differential Melanopic Photic}

\section{Stimulation in Humans}

Shao-Min Hung ${ }^{1 \mp{ }^{1 *}}$, Dan Milea ${ }^{1-3 \pi}$, Annadata Venkata Rukmini ${ }^{1}$, Raymond P. Najjar ${ }^{3}$, Joo Huang Tan ${ }^{1}$, Françoise Viénot $^{4}$, Marie Dubail ${ }^{4}$, Sharon Lee Choon Tow ${ }^{1-3.5}$, Tin Aung ${ }^{1-3}$, Joshua J. Gooley ${ }^{1}$, Po-Jang Hsieh ${ }^{1 *}$

${ }^{1}$ Neuroscience and Behavioral Disorders Program, Duke-NUS Medical School, Singapore,

${ }^{2}$ Singapore National Eye Centre, Singapore,

${ }^{3}$ Singapore Eye Research Institute, Singapore,

${ }^{4}$ Centre de recherche sur la conservation (CRC, USR 3224), Sorbonne Universités, Muséum national d'Histoire naturelle, Ministère de la Culture et de la Communication, CNRS, France,

${ }^{5}$ National University of Singapore, Singapore

${ }^{\pi}$ Co-first-authors

*Corresponding authors: Shao-Min Hung shaomin.hung@u.duke.nus.edu \& Po-Jang Hsieh pojang.hsieh@duke-nus.edu.sg, Neuroscience and Behavioral Disorders Program, Duke-NUS Medical School, 8 College Road, Singapore 169857

Key words: Melanopsin, intrinsically photoreceptive retinal ganglion cells, frontal eye field, blue light, metamers, magnetic resonance imaging 


\begin{abstract}
Photic stimulation of rods, cones and intrinsically photosensitive melanopsin-containing retinal ganglion cells (ipRGCs) mediates non-visual light responses, including entrainment of circadian rhythms and pupillary light reflex. Unlike visual responses to photic stimulation, the cerebral correlates of non-visual light responses in humans remains elusive. In this study, we used functional magnetic resonance imaging (fMRI) in 14 healthy young participants, to localize cerebral regions which are differentially activated by metameric light, which gave rise to different levels of melanopic excitation. Mean blood oxygen-level dependent (BOLD) responses disclosed bilateral activation of the frontal eye fields during exposure to light geared towards melanopsin. Furthermore, multivariate pattern analyses showed distinct bilateral pattern activity in the inferior temporal gyri and the caudate nuclei. Taken together, our findings suggest that melanopsin-based photoreception activates a cerebral network including frontal regions, classically involved in attention and ocular motor responses.
\end{abstract}




\section{Introduction}

In addition to the classical rod and cone photoreceptors in the outer retina, a small subset of retinal ganglion cells contain the photopigment melanopsin and respond directly to light (Berson, 2003; Dacey et al., 2005; Gooley et al., 2001; LeGates et al., 2014; Provencio et al., 1999). These intrinsically photosensitive retinal ganglion cells (ipRGCs) respond preferentially to short wavelength blue light, but also integrate neural signals originating from rods and cones, before projecting to various cerebral structures implicated in non-visual responses to light such as the pupillary light reflex and circadian entrainment (Lucas et al., 2014). Recent studies suggest that ipRGCs may contribute to luminance differentiation in blind patients without functional rods and cones (Zaidi et al., 2007) and brightness detection in healthy individuals (Brown et al., 2012). Although the response properties of ipRGCs and their efferent projections have been thoroughly investigated in animal models (Hatori and Panda, 2010; LeGates et al., 2014), little is known regarding their cerebral correlates in humans. This is mainly due to methodological limitations in vivo and the complexity of identifying brain activations specifically mediated by the intrinsic (melanopic) stimulation of ipRGCs from those originating from classical visual photoreceptors (Vandewalle et al., 2013; 2009; 2007b). To date, a handful of studies have used narrow-bandwidth light stimuli and functional magnetic resonance imaging (fMRI) to investigate non-visual lightinduced cerebral activations in humans (Chellappa et al., 2014; Perrin et al., 2004; Vandewalle et al., 2007b; 2007a). For example, using complex tasks such as working memory task, both Chellappa et al. (2014) and Vandewalle et al. (2007b) show that executive regions may be susceptible to wavelength-dependent influence of light exposure. These studies could not fully isolate melanopsindependent cerebral activations, however, because the light stimuli that were used presumably activated both ipRGCs and visual photoreceptors. In order to isolate the intrinsic photo-responses of ipRGCs, we designed two perceptually similar white lights (i.e., metameric light stimuli) with different levels of melanopic excitation and similar stimulation of cones (Estévez and Spekreijse, 1982; Estévez and Spekreuse, 1974; Viénot and Brettel, 2014). By combining individually tailored 
metameric light stimulation and fMRI, we explored the cerebral correlates of ipRGC photic activation in healthy individuals.

\section{Methods}

\section{Participants}

Seventeen healthy participants (18-35 years) took part in a study that included both psychophysical and fMRI assessments. All participants were free of any neurological, psychiatric or sleep disorders and were not on any medication; they all had normal visual functions and normal color vision assessed by Ishihara's test for Color-Blindness. Participants were instructed to keep regular sleep cycle a week before the experiment and refrain from consuming alcohol and caffeine 24 hours prior to the experiment. Also, according to a follow-up report, none of the participants reported having any sleep issues. The experiments conducted complied with the Helsinki tenets for biomedical research and were approved by the institutional review boards at the National University of Singapore and Singapore Health Services. All participants gave written informed consent prior to the experiments. Three participants were excluded from data analysis: one participant with excessive head movements and two participants with corrupted data. Fourteen participants (5 males, 2 left handed, 1 smoker, mean age \pm SD was $24.7 \pm 4.5$ years, average BMI was $20.2 \pm 2.3$.) were included in the final analysis. According to post-experiment follow-up questionnaires, none of the participants reported having any sleep issues. Participants were scanned during daytime (i.e. 9 am $12 \mathrm{pm} \& 2-5 \mathrm{pm}, \mathrm{n}=5$ ) and nighttime (i.e. $7-10 \mathrm{pm}, \mathrm{n}=9$ ).

\section{Metameric lights generation}

LED projector and calibration 
A light projector comprising 60 light-emitting-diodes (LEDs) of 7 color types was used in the current study (Desire D60, Electronic Theatre Controls, Middleton, Wisconsin, U.S.). The spectral radiance of the lights transmitted was measured on a colorless diffuser (Canson, Annonay, France), which was also used in our later psychophysical and fMRI experiments. The measurements were obtained by a JETI specbos 1211 UV spectroradiometer (JETI, Jena, Germany) (see (Viénot et al., 2012) for calibration details).

Photic stimulations

We created a pair of metameric photic stimulations that excited cones similarly but have either a high (HM) or low (LM) melanopsin stimulation. These lights were first generated from standard fundamental observers (CIE, 2006; Stockman and Sharpe, 2000; Viénot et al., 2012) and later tailored to each participant in our psychophysical experiment. The mean melanopic excitation difference was $49.9 \pm 10.4 \%$ (mean $\pm \mathrm{SD}$ ). Please refer to supplementary information for light production details. We have also provided a table of HM/LM melanopsin stimulations in arbitrary units for each participant (Table S. 1.).

\section{Psychophysical refinement of the photic stimulations}

\section{Design}

The psychophysical experiment was performed in the MRI environment and was designed to tailor the metameric light stimuli for each participant. Participants were exposed to photic stimulation monocularly (left eyes) throughout the psychophysical and fMRI experiments, to avoid potential differences in color perception between the two eyes. Their pupils were not dilated with medication. 
The photic stimuli were projected on a diffuser fixed $5 \mathrm{~cm}$ in front of the eyes of the participant (via a headmounted mask). During this procedure, participants were fixating a central fixation area (subtending central 10 degrees), which was designed to reduce the influence of the Maxwell's spot (Horiguchi et al., 2013). Thus, participants could perceive a homogenous light distribution within their whole visual field around the fixation point.

The following two steps were implemented to generate the individually-tailored HM/LM pair. We used flicker photometry to refine the perceptual match of the HM/LM pair in each participant. In this paradigm, the two lights were shown in a successive manner at least 3 times. Each time each light was presented for approximately 3 seconds with no blank interval in-between the two lights. The initial luminance level of the lights were similar to that of the final light stimuli except that the final light pairs had smaller luminance difference due to $25 \mathrm{~Hz}$ photometry adjustment (see below). Participants were instructed to report any perceptual differences (e.g. the $1^{\text {st }}$ light was greener). Based on the participant's feedback the experimenter adjusted the light channels without changing the "blue" channel. This was to ensure sufficient melanopic excitation differences. The color matching procedure stopped when a.) participants reported seeing no color differences between the two lights or b.) participants reported seeing minimal color differences that couldn't be diminished. Subsequently to this initial step, we created 11 pairs of light with varied luminance level in one of the lights (i.e. \pm 5 units alpha value). Participants were then asked to choose the pair with least flickering sensation when presented at $25 \mathrm{~Hz}$. We repeated this procedure twice to find the pair that produced minimal or no flickering sensation. Finally, the participants were asked to rate the color differences between the two lights in the finalized HM/LM pair and 27 other EM pairs, without knowing the identities of all the pairs. The EM pairs that had the same ratings as the HM/LM pair were chosen as the controls. 
Despite individually tailoring the lights to each observer, residual color perception differences remained between HM and LM metamers. To examine whether this minimal color difference between the metameric HM/LM pair might elicit differential brain activation and confound the results, an additional control experiment was performed. This control experiment was implemented to select pairs of light with equal melanopic stimulation (EM) that simulated the perceived color difference between the HM/LM pair. Each participant was then asked to rate the color differences (with a 7-point scale) of the above individually-tailored HM/LM pair among 27 EM pairs, which were also close-to-white lights, without knowing the identities of all these pairs. At least 2 (maximum = 3) EM pairs that had the same mean color difference rating score as that of the HM/LM pair were chosen as our control pairs (mean ratings for HM/LM pairs and EM pairs, respectively: 3.5 vs. $3.5, p>0.05)$. The two lights in each EM pair had equal luminance and the same melanopic excitation level. (Please refer to supplementary information for details of the EM pairs.) If the control pairs with the same minimal color difference as the HM/LM pair do not reveal any differential brain activation patterns, then it makes the color difference unlikely to have significantly contributed to the observed effect. The spectral radiance of a representative HM/LM and EM pairs is shown in Fig. 1.

\section{fMRI experiment}

\section{Design}

Scanning was performed using a 3T Siemens Trio scanner (Siemens, Erlangen, Germany) at Duke-NUS Medical School, Singapore. Functional MRI runs were acquired using a gradient echo-planar imaging sequence $(\mathrm{TR}=2 \mathrm{~s}$, TE $30 \mathrm{~ms}$, FA 75, FOV $192 \times 192 \mathrm{~mm}, 64 \times 64$ matrix, $3 \times 3 \mathrm{~mm}$ in-plane resolution). Thirty-six slices were collected with a 12-channel head coil (3.8 $\mathrm{mm}$ thickness). Slices were oriented roughly parallel to the AC-PC with whole brain covered. A T1-weighted anatomical image was 
also acquired and later used for co-registration (TR = 2.3 s, TI $900 \mathrm{~ms}$, flip angle 9, $\mathrm{BW} 240 \mathrm{~Hz} /$ pixel, FOV $256 \times 240 \mathrm{~mm}, 256 \times 256$ matrix, 192 slices, $1 \times 1 \times 1 \mathrm{~mm}$ ). Each participant took part in 12 runs, half of which (HM/LM runs) contained blocks of HM and LM lights and the other half (EM runs) contained blocks of EM lights. Each run contained 9 blocks and followed an alternating light off - light on procedure. Each block lasted $30 \mathrm{~s}$ with a counterbalanced presentation order of the HM and LM lights or with a counterbalanced presentation order of the 2 lights in the EM pairs (Fig. 2). The setup in the light off and light on blocks was identical, except that all light sources in the scanner environment were eliminated in the light off condition. Participants were required to fixate at the center of a black disc with their left eyes. Three additional runs of retinotopic mapping were collected to localize striate and extrastriate cortex. Retinotopic mapping scans consisted of six 20-second blocks each flanked by 20-second fixation. Stimuli were presented in three experimental conditions. Each condition was repeated twice in a single run. The conditions were presented in a pseudo-randomised order across all three blocks. In the retinotopic mapping scans, flashing checkerboard wedges were presented in each condition. In the horizontal condition, two wedges subtending 10 degrees from the central fixation were presented along the horizontal meridian. Similarly, in the vertical condition, the two wedges were presented along the vertical meridian. In the last experimental condition, four wedges each subtending 30 degrees from fixation were presented along the diagonal axis. During a stimulus block, color of the central fixation changed between green and red. Subjects were tasked to maintain fixation at all times and indicate color of central fixation cross via button presses.

Data analysis

fMRI data analysis was conducted using freesurfer (http://surfer.nmr.mgh.harvard.edu/) and MATLAB (MathWorks). The processing steps for both the retinotopic mapping and the experimental runs included motion correction and linear trend removal. The processing for the retinotopic mapping runs and experimental runs used for later univariate (mean) BOLD responses 
analysis also included spatial smoothing with a 6-mm kernel. For every participant, all the runs were modeled with general linear model (GLM). A gamma function with delta $(\delta)=2.25$ and tau $(\tau)$ $=1.25$ was used to estimate the hemodynamic response for each condition in the retinotopic mapping scans and the experimental runs.

Univariate (mean) BOLD responses

The mean response in each voxel ( $\beta$ value) was extracted and compared between HM and LM photic stimulations. A group-level two-tailed one-sample t-test was performed across all participants on each voxel to find regions that have stronger mean BOLD responses while viewing HM lights, compared to viewing LM lights. The resulting statistical maps were corrected with cluster-thresholding ( $p<0.005$; cluster-forming threshold $p<0.01)$.

Searchlight-based multivariate pattern analyses

Whole-brain searchlight multivariate (multivoxel) pattern analysis (MVPA) was performed after Talairach spatial standardization. Two different sizes of searchlight cubes centered on the original voxel were defined. A $7 \times 7 \times 7$ voxels cube was used in the whole-brain search; another $3 \times 3 \times 3$ voxels searchlight cube was also implemented, aiming to identify significant pattern information differences in small structures (e.g. subcortical regions). For normalization, the mean response in each voxel ( $\beta$ value) in the two light conditions was subtracted from the response to individual light condition before calculating the correlations. Data were split into odd and even runs, and activation patterns were extracted from each subset of data, namely HM_odd, HM_even, LM_odd, and LM_even. We then computed the Pearson correlation coefficients between the activation patterns of two light conditions (Haxby et al., 2001). These correlations were computed individually and later 
averaged across participants by condition (HM and LM). In every selected region deemed to contain discriminative activation pattern information for the two light stimuli, the pattern response across voxels in that region produced by the same light would be more similar than that produced by two different lights, i.e. $\Delta \mathrm{r}\left(=\mathrm{r}_{\text {within condition }}-\mathrm{r}_{\text {between conditions }}\right)>0$. A Fisher-Z transformation was computed on each correlation map, and the resultant z-map was then applied a Gaussian smoothing function with an estimated full-width at half maximum of $6 \mathrm{~mm}$. A group-level one-tailed onesample t-test was performed across all participants on each voxel to test whether any region contained significant pattern information. The resulting statistical maps were corrected with clusterthresholding (Hagler et al., 2006) ( $p<0.005$; cluster- forming threshold $p<0.01$ ).

\section{Results}

\section{fMRI experiment}

\section{Mean BOLD responses}

Bilateral frontal eye fields (FEF) were associated with stronger BOLD responses during the contrast HM versus LM (Fig. 3; Table 1). No mean activation differences were found in the EM control pairs. This bilateral FEF finding was further replicated by a leave-one-out cross-validation ROI analysis $(p<.05$; see supplementary information for analysis details.).

Pattern information

Multivariate pattern analysis with a small $3 \times 3 \times 3$ searchlight cube, which was designed to explore small brain regions, found distinctive response patterns in bilateral caudate nucleus (Fig. 4; Table 2). 
Using the same contrast (HM versus LM) and the same multivariate pattern analysis, a larger $7 \times 7$ $\mathrm{x} 7$ searchlight cube showed activation of the right medial frontal gyrus and orbitofrontal cortex, left medial frontal gyrus and occipital cortex, bilateral inferior temporal gyrus (ITG), and cerebellum (Fig. 5; Table 3). No region showed significant pattern activation differences in the EM control pairs. Comparisons of results in participants recruited during daytime (i.e. before $5 \mathrm{pm}, \mathrm{n}=5$ ) and nighttime (i.e. after $7 \mathrm{pm}, \mathrm{n}=9$ ), did not disclose any significant difference in either univariate or multivariate analysis. We acknowledge that we might not have enough statistical power to observe the difference. Further investigations on the rhythmicity of melanospin-mediated cerebral responses are required.

Retinotopic mapping

None of the 7 regions selected (i.e. V1_all /V1_right hemisphere (rh) /V1_left hemisphere (lh)/

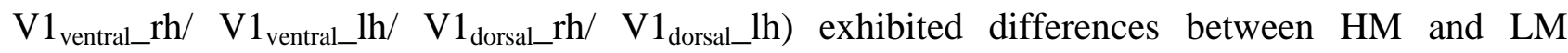
stimulations, both in univariate mean BOLD and multivariate pattern analyses.

Leave-one-out cross-validation ROI analysis

To further validate our bilateral FEF finding, another univariate mean BOLD responses analysis was performed on 13 out of 14 participants. The mean response in each voxel ( $\beta$ value) was extracted for [HM], [LM], [EM1] and [EM2] photic stimulations. A double contrast comparison was made (i.e. $\left.\beta_{\{[\mathrm{HM}]-[\mathrm{LM}]\}}-\beta_{\{([\mathrm{EM} 1]-[\mathrm{EM} 2])\}}\right)$, and a group-level one-tailed one-sample t-test was performed across 13 participants on the whole brain. The bilateral FEF was identified functionally as a ROI with the resultant mean BOLD responses map (corrected with cluster-thresholding $(p<$ 0.01; cluster- forming threshold $p<0.05)$ ). After the ROI was defined, the mean response in each 
voxel ( $\beta$ value) within the ROI for [HM], [LM], [EM1] and [EM2] in the left-out participant was extracted. We reiterated this procedure for 14 times and the group $\beta$ values in the four conditions were plotted in Figure 6. A group-level one-tailed one-sample t-test was performed on the group differences (i.e. $\beta_{\{[\mathrm{HM}]-[\mathrm{LM}]\}}$ V.s. $\left.\beta_{\{([\mathrm{EM} 1]-[\mathrm{EM} 2])\}}\right)$ with a $t(13)=1.85, p<.05$ (Fig. 6).

\section{Discussion}

In conclusion, this study brings support for a novel involvement of melanopsin-mediated photoreception in the activation of several cortical and sub-cortical areas. The main findings of the study are that ipRGC-specific photoreception may be involved in the activation of the cortical frontal eye fields, and modulates the activation of the inferior temporal gyri as well as other subcortical regions (i.e. caudate nuclei).

Herein, we show that greater melanopsin stimulation is associated with stronger mean BOLD responses in the bilateral frontal eye fields (FEFs). Our fMRI results suggest that a photic stimulation of the ipRGCs enhances FEF activation. The FEFs are involved in the cortical control of eye movements (Milea et al., 2002; 2005; Jamadar, 2013), visual selection (Moore and Armstrong, 2003; Muggleton, 2003), covert attention (Buschman and Miller, 2009), ocular motor decisions (Milea et al., 2007), and top-down anticipatory control of the visual system (Marshall et al., 2015). These cerebral regions act as primers for cortical processes since their preactivation improves visual detection through a top-down modulation of the excitability within the extra-striate visual cortex (Silvanto, 2006). The FEF has also been implicated in covert attention, and hence it is possible that melanopsin modulates alertness/attention (Martinez-Conde et al., 2004). This interpretation is consistent with the proposed involvement of melanopsin in increasing vigilance (Lockley et al. 2006). Future experiments are required to carefully examine these possibilities: examining the contribution of melanopsin in eye movements and/or attention. 
In a multivariate pattern analysis designed to investigate the responses of smaller brain structures, we showed distinct activation patterns of the bilateral caudate nuclei. These nuclei could be involved in the visual perception-action network, receiving inputs from the FEFs and modulating motor superior colliculus activity via the substantia nigra for saccade generation (Ding and Gold, 2012; Opris et al., 2013). Additionally, the dedicated MVPA showed that lights with different levels of melanopic excitation induced different activation patterns in the bilateral ITG that also receives inputs from the FEF (Baizer et al., 1991), in addition to being the terminal region of the ventral visual pathway. Finally, our paradigm also disclosed that the photic stimulation of ipRGCs is associated with cerebellar activation, which has been implicated in the control of eye movements, both in humans and in animals (Robinson and Fuchs, 2001; Stanton et al., 1988). Together, these findings suggest that the ipRGCs photoreception may be involved in ocular motor preparation or execution. It is worth noting that our retinotopic mapping did not disclose early visual cortex activation, which is consistent with the results of a recent study showing no significant visual cortex response to light stimulations targeting melanopsin (Spitschan et al., 2016). These findings suggest that ipRGCs photoreception may bypass early visual cortices and connect to FEF and ITG via subcortical pathways.

Since the task in our study was not cognitively demanding (fixating a dot), we cannot exclude that a more demanding task that requires wider brain network activation could have induced a broader range of differential melanopic brain activation. Vandewalle et al. (2007b) scanned healthy participants stimulated with 3 monochromatic lights while performing a working memory task. An increased brain activity in the hippocampus, thalamus and amygdala was found under blue light compared to green light stimulation. Other regions including middle frontal gyrus, thalamus and brainstem were also more activated under blue light compared to violet light. These findings suggest that exposure to blue light and direct ipRGC stimulation could trigger widespread brain 
activation. However, perceptual differences of distinct monochromatic lights could have been be a confounding factor in these results, and the study design could not distinguish between activation of melanopsin versus S-cones. Future experiments in sighted individuals should control for both perceptual differences in light stimulations (e.g., the silent substitution approach used in the current study) and include more demanding tasks that might recruit additional brain networks. In a subsequent study, Vandewalle et al. (2013) recruited three blind patients with no light perception who were exposed to blue light while performing an auditory working memory task. The authors again found widespread brain activation when comparing the effects of blue light to darkness. These activations included the ventrolateral prefrontal cortex, precuneus, and anterior cingulate cortex, which are components of the so-called default mode network, suggesting that ipRGCs may be involved in a wider brain network activation. Nonetheless, it is not clear whether other monochromatic lights would trigger the same effect, and whether the results were affected by these patients' pathology.

The study however, has a few limitations that should be reported. First, even though participants were instructed to maintain a stable fixation, we reckon that using an eye-tracking system would have been optimal to ensure compliance to protocol. Unfortunately, it was impossible to accommodate the system without interfering with the light projection. Therefore, we cannot exclude that the FEF activation associated with melanopsin stimulation could be due to a greater number of fixational eye movements, to eyelid movements due to photophobia, or to increased engagement of attentional mechanisms. Second, unlike previous animal studies in mice lacking rods and cones (e.g. Lucas, et al., 1999), a direct and isolated stimulation of melanopsin in humans is not achievable to date. Even though the metameric lights we used in this study were carefully designed to silence cones and differentially activate melanopsin, and have been functionally verified in a previous study in humans using pupillary responses (Viénot et al., 2012), we cannot fully exclude minor differential rod and cone intrusions. 
In conclusion, this study brings forth yet a novel involvement of melanopsin-mediated photoreception in the activation of several cortical and sub-cortical areas. The stronger correlation between FEF and metameric stimulation of melanopsin suggests that ipRGC photoreception might activate FEF that have been implicated in attention, eye movement, and other ocular motor functions. 


\section{Acknowledgements}

This work was supported by the SNEC Health Research Endowment (HREF) grant R1040/55/2013.

We thank Rene Muri and John Barbur for their important comments and suggestions. We also thank the three anonymous reviewers for their comments and extensive reviews, which have helped improve the clarity of the paper. 


\section{References}

Baizer, J.S., Ungerleider, L.G., Desimone, R., 1991. Organization of visual inputs to the inferior temporal and posterior parietal cortex in macaques. The Journal of Neuroscience 11, 168-190.

Berson, D., 2003. Strange vision: ganglion cells as circadian photoreceptors. Trends in Neurosciences 26 , 314-320.

Brown, T.M., Tsujimura, S.-I., Allen, A.E., Wynne, J., Bedford, R., Vickery, G., Vugler, A., Lucas, R.J., 2012. Melanopsin-Based Brightness Discrimination in Mice and Humans. Current Biology 22, 11341141.

Buschman, T.J., Miller, E.K., 2009. Serial, Covert Shifts of Attention during Visual Search Are Reflected by the Frontal Eye Fields and Correlated with Population Oscillations. Neuron 63, 386-396.

Chellappa, S.L., Ly, J.Q.M., Meyer, C., Balteau, E., Degueldre, C., Luxen, A., Phillips, C., Cooper, H.M., Vandewalle, G., 2014. Photic memory for executive brain responses. Proceedings of the National Academy of Sciences 111, 6087-6091.

CIE, 2006. Fundamental Chromaticity Diagram with Physiological Axes - Part 1.

Dacey, D.M., Liao, H.-W., Peterson, B.B., Robinson, F.R., Smith, V.C., Pokorny, J., Yau, K.-W., Gamlin, P.D., 2005. Melanopsin-expressing ganglion cells in primate retina signal colour and irradiance and project to the LGN. Nature 433, 749-754.

Ding, L., Gold, J.I., 2012. Separate, Causal Roles of the Caudate in Saccadic Choice and Execution in a Perceptual Decision Task. Neuron 75, 865-874.

Estévez, O., Spekreijse, H., 1982. The "silent substitution" method in visual research. Vision Research 22, 681-691.

Estévez, O., Spekreuse, H., 1974. A spectral compensation method for determining the flicker characteristics of the human colour mechanisms. Vision Research 14, 823-830.

Gooley, J.J., Lu, J., Chou, T.C., Scammell, T.E., Saper, C.B., 2001. Melanopsin in cells of origin of the retinohypothalamic tract. Nat Neurosci 4, 1165-1165.

Hagler, D.J., Saygin, A.P., Sereno, M.I., 2006. Smoothing and cluster thresholding for cortical surfacebased group analysis of fMRI data. NeuroImage.

Hatori, M., Panda, S., 2010. The emerging roles of melanopsin in behavioral adaptation to light. Trends in Molecular Medicine 16, 435-446.

Haxby, J.V., Gobbini, M.I., Furey, M.L., Ishai, A., Schouten, J.L., Pirtrini, P., 2001. Distributed and overlapping representations of faces and objects in ventral temporal cortex. Science 293, 2425-2430.

Horiguchi, H., Winawer, J., Dougherty, R.F., Wandell, B.A., 2013. Human trichromacy revisited. Proceedings of the National Academy of Sciences 110, E260-E269.

Jamadar, S.D., 2013. Quantitative meta-analysis of fMRI and PET studies reveals consistent activation in fronto-striatal-parietal regions and cerebellum during antisaccades and prosaccades 1-15.

Langel, J.L., Smale, L., Esquiva, G., Hannibal, J., 2015. Central melanopsin projections in the diurnal rodent, Arvicanthis niloticus. Front. Neuroanat. 9.

LeGates, T.A., Fernandez, D.C., Hattar, S., 2014. Light as a central modulator of circadian rhythms, sleep and affect. Nature reviews neuroscience 15, 443-454.

Lennie, P., Pokorny, J., Smith, V.C., 1993. Luminance. JOSA A 10, 1283-1293.

Lockley, S.W., Evans, E.E., Scheer, F., 2006. Short-wavelength sensitivity for the direct effects of light on alertness, vigilance, and the waking electroencephalogram in humans. SLEEP 29, 161-168.

Lucas, R.J., Freedman, M.S., Muñoz, M., Garcia-Fernández, J.-M., Foster, R.G., 1999. Regulation of the mammalian pineal by non-rod, non-cone, ocular photoreceptors. Science 284, 505-507.

Lucas, R.J., Peirson, S.N., Berson, D.M., Brown, T.M., Cooper, H.M., Czeisler, C.A., Figueiro, M.G., Gamlin, P.D., Lockley, S.W., O’Hagan, J.B., Price, L.L.A., Provencio, I., Skene, D.J., Brainard, G.C., 2014. Measuring and using light in the melanopsin age. Trends in Neurosciences 37, 1-9.

Marshall, T.R., O'Shea, J., Jensen, O., Bergmann, T.O., 2015. Frontal Eye Fields Control Attentional 
Modulation of Alpha and Gamma Oscillations in Contralateral Occipitoparietal Cortex. Journal of Neuroscience 35, 1638-1647.

Martinez-Conde, S., Macknik, S.L., Hubel, D.H., 2004. The role of fixational eye movements in visual perception. Nature reviews neuroscience 5, 229-240.

Milea D, Lobel E, Lehéricy S, Pierrot-Deseilligny C, Berthoz A., 2005. Cortical mechanisms of saccade generation from execution to decision. Ann N Y Acad Sci 1039, 232-238.

Milea D, Lobel E, Lehéricy S, Duffau H, Rivaud-Péchoux S, Berthoz A, Pierrot-Deseilligny C., 2002. Intraoperative frontal eye field stimulation elicits ocular deviation and saccade suppression. Neuroreport 13, 1359-64.

Milea, D., Lobel, E., Lehéricy, S., Leboucher, P., 2007. Prefrontal cortex is involved in internal decision of forthcoming saccades. Neuroreport 18, 1221-1224.

Moore, T., Armstrong, K.M., 2003. Selective gating of visual signals by microstimulation of frontal cortex. Nature 421, 370-373.

Muggleton, N.G., 2003. Human Frontal Eye Fields and Visual Search. Journal of Neurophysiology 89, 3340-3343.

Opris, I., Santos, L., Gerhardt, G.A., Song, D., Berger, T.W., Hampson, R.E., Deadwyler, S.A., 2013. Prefrontal cortical microcircuits bind perception to executive control. Scientific Reports 3.

Perrin, F., Peigneux, P., Fuchs, S., Verhaeghe, S., Laureys, S., Middleton, B., Degueldre, C., Del Fiore, G., Vandewalle, G., Balteau, E., Poirrier, R., Moreau, V., Luxen, A., Maquet, P., Dijk, D.-J., 2004. Nonvisual Responses to Light Exposure in the Human Brain during the Circadian Night. Current Biology 14, 1842-1846.

Provencio, I., Rodriguez, I.R., Jiang, G., Hayes, W.P., Moreira, E.F., Rollag, M.D., 1999. A novel human opsin in the inner retina. The Journal of Neuroscience 20, 600-605.

Robinson, F.R., Fuchs, A.F., 2001. The role of the cerebellum in voluntary eye movements. Annual review of neuroscience 24, 981-1004.

Schall, J.D., Hanes, D.P., 1993. Neural basis of saccade target selection in frontal eye field during visual search. Nature 366, 6454.

Schall, J.D., Hanes, D.P., Thompson, K.G., 1995. Saccade target selection in frontal eye field of macaque. I. Visual and premovement activation. The Journal of Neuroscience 15, 6905-6918.

Silvanto, J., 2006. Stimulation of the Human Frontal Eye Fields Modulates Sensitivity of Extrastriate Visual Cortex. Journal of Neurophysiology 96, 941-945.

Spitschan, M., Datta, R., Stern, A.M., Brainard, D.H., Aguirre, G.K., 2016. Human Visual Cortex Responses to Rapid Cone and Melanopsin-Directed Flicker. Journal of Neuroscience 36, 1471-1482.

Stanton, G.B., Goldberg, M.E., Bruce, C.J., 1988. Frontal eye field efferents in the macaque monkey: II. Topography of terminal fields in midbrain and pons. Journal of Comparative Neurology 271, 493506.

Stockman, A., Sharpe, L.T., 2000. The spectral sensitivities of the middle-and long-wavelength-sensitive cones derived from measurements in observers of known genotype. Vision Research 40, 1711-1737.

Vandewalle, G., Collignon, O., Hull, J.T., Daneault, V., Albouy, G., Lepore, F., Phillips, C., Doyon, J., Czeisler, C.A., Dumont, M., 2013. Blue light stimulates cognitive brain activity in visually blind individuals. Journal of Cognitive Neuroscience 25, 2072-2085.

Vandewalle, G., Gais, S., Schabus, M., Balteau, E., Carrier, J., Darsaud, A., Sterpenich, V., Albouy, G., Dijk, D.J., Maquet, P., 2007a. Wavelength-Dependent Modulation of Brain Responses to a Working Memory Task by Daytime Light Exposure. Cerebral Cortex 17, 2788-2795.

Vandewalle, G., Maquet, P., Dijk, D.-J., 2009. Light as a modulator of cognitive brain function. Trends in Cognitive Sciences 13, 429-438.

Vandewalle, G., Schmidt, C., Albouy, G., Sterpenich, V., Darsaud, A., Rauchs, G., Berken, P.-Y., Balteau, E., Degueldre, C., Luxen, A., Maquet, P., Dijk, D.-J., 2007b. Brain Responses to Violet, Blue, and Green Monochromatic Light Exposures in Humans: Prominent Role of Blue Light and the Brainstem. PLoS ONE 2, e1247.

Viénot, F., Brettel, H., Dang, T.-V., Le Rohellec, J., 2012. Domain of metamers exciting intrinsically 
photosensitive retinal ganglion cells (ipRGCs) and rods. JOSA A 29, A366-A376.

Viénot, F., Brettel, H., 2014. The Verriest Lecture: Visual properties of metameric blacks beyond cone vision. JOSA A 31, A38-A46.

Zaidi, F.H., Hull, J.T., Peirson, S.N., Wulff, K., Aeschbach, D., Gooley, J.J., Brainard, G.C., GregoryEvans, K., Rizzo, J.F., III, Czeisler, C.A., 2007. Short-wavelength light sensitivity of circadian, pupillary, and visual awareness in humans lacking an outer retina. Current Biology 17, 2122-2128. 